\title{
Conocimientos matemáticos de los estudiantes de primer ingreso del CUValles de la Universidad de Guadalajara
}

\author{
Mathematical Knowledge of First Entry Students of the Centro Universitario \\ de los Valles of the Universidad de Guadalajara
}

Conhecimento matemático de alunos iniciantes no CUValles na Universidade de Guadalajara

Silvia Sánchez Díaz Universidad de Guadalajara, Centro Universitario de los Valles, México diazsilvia09@hotmail.com https://orcid.org/0000-0002-4790-0503

Emilio Leonardo Ramírez Mora Universidad de Guadalajara, Centro Universitario de los Valles, México emiliorm@valles.udg.mx https://orcid.org/0000-0001-9405-5392

César Calderón Mayorga Universidad de Guadalajara, Centro Universitario de los Valles, México cesarcm@valles.udg.mx https://orcid.org/0000-0003-4216-1219

Rubén Sánchez Gómez Universidad de Guadalajara, Centro Universitario de Ciencias Exactas e Ingenierías, México dr.rsanchez@gmail.com https://orcid.org/0000-0002-1103-3436 


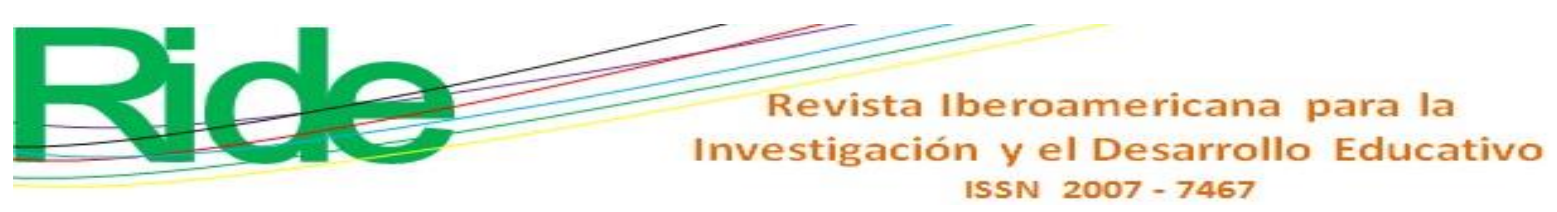

\section{Abstract}

In this research, we analyze the knowledge in mathematics that have the first entry students of the Bachelor of Administration, Tourism, Public Accounting, Information Technology and Engineering in Mechatronics, Electronics and Computation, Electronic Instrumentation and Nanosensors, Molecular Design of Materials, Geophysics and Biological Systems. The goal is to identify the basic knowledge and the deficiencies of the students in this area before taking subjects such as Mathematics I or Pre-calculus (according to the case), so that these deficiencies do not contribute to failure, backwardness and possible abandonment of undergraduate studies. A test consisting of 15 multiple choice questions was applied to 413 students of these educational programs. As part of the analysis, it was found $44.20 \%$ of incorrect answers, $52.98 \%$ of correct answers and $2.82 \%$ of unanswered answers. An average score of 52.97 (failing) was obtained; only 3 students correctly answered the 15 questions and one student got a correct answer of the 15. The item 13, which requires to express algebraically the perimeter of a rectangle from a verbal expression, was the one that obtained the highest number of incorrect answers with $11.56 \%$. Question 14, which consists in identifying the procedure to solve a first degree equation with an unknown, was the one that obtained the highest number of correct answers with $10.69 \%$. The educational programs that obtained an average higher than the general average (52.97) were Mechatronics Engineering, Electronic Instrumentation and Nanosensors, Molecular Design of Materials, Geophysics and the Bachelor of Public Accounting; the rest had an average below the general average. Only Mechatronic Engineering and Geophysical Engineering students obtained passing grades averages. It is necessary that all bachelor's degrees correct these gaps, relying on the courses of Mathematics I and Precalculus. It is necessary to strength knowledge of fractions, exponents, hierarchy of operations, law of signs, and algebraic operations.

Keywords: mathematics knowledge, higher education, first entry students, educational program, test. 


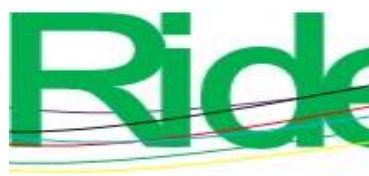

Revista Iberoamericana para la Investigación y el Desarrollo Educativo ISSN $2007-7467$

\section{Resumo}

Esta pesquisa analisa os conhecimentos matemáticos dos alunos do primeiro ano do Bacharelado em Administração, Turismo, Contabilidade Pública, Tecnologia da Informação e engenharia em Mecatrônica, Eletrônica e Computação, Instrumentação Eletrônica e Nanossensores, Projeto Molecular de Materiais, Geofísica e Sistemas Biológicos. O objetivo é identificar o conhecimento básico e as deficiências dos alunos nessa área antes de cursar matérias como Matemática I ou Pré-cálculo (conforme o caso) para ajudar a erradicar o fracasso, o atraso e o possível abandono dos estudos de graduação. Um teste composto por 15 itens de múltipla escolha foi aplicado a 413 estudantes dos referidos programas educacionais. Após a análise, foram encontrados 44,20\% de respostas incorretas, 52,98\% de respostas corretas e 2,82\% de respostas não respondidas. Foi obtida uma nota média de 52,97 (reprovada); apenas três alunos responderam os 15 itens corretamente; um aluno correspondeu a apenas um do número total de itens. O reagente 13 , em que é necessário expressar algebricamente o perímetro de um retângulo a partir de uma expressão verbal, obteve o maior número de respostas incorretas, com $11,56 \%$. O reagente 14 , que consiste em identificar o procedimento para resolver uma equação de primeiro grau com uma variável desconhecida, registrou o maior número de respostas corretas, com 10,69\%. Os programas que obtiveram média mais alta que a média geral $(52,97)$ foram de engenharia em mecatrônica, instrumentação eletrônica e nanossensores, design de materiais moleculares, geofísica e graduação em contabilidade pública; o restante ficou abaixo da média geral. Somente na engenharia mecatrônica e na engenharia geofísica foram obtidas as médias das notas de aprovação. Pelo exposto, é necessário que essas deficiências sejam corrigidas em todos os graus, contando com os cursos de Matemática I e Pré-cálculo, e especificamente é necessário fortalecer o conhecimento em frações, expoentes, hierarquia de operações, lei de sinais e operações algébricas.

Palavras-chave: conhecimento matemático, ensino superior, alunos da primeira entrada, programa educacional, teste.

Fecha Recepción: Enero 2020

Fecha Aceptación: Mayo 2020 


\section{Introducción}

El Centro Universitario de los Valles (CUValles) de la Universidad de Guadalajara brinda educación de nivel superior en la región de los Valles del estado de Jalisco, México. Tiene dos ciclos de ingreso de estudiantes a sus distintas licenciaturas: el primero de ellos en enero (ciclo A) y el segundo en agosto (ciclo B).

Los estudiantes provienen principalmente de distintos bachilleratos de la región en la que se ubica el centro universitario, tal y como preparatorias regionales, módulos y extensiones de la Universidad de Guadalajara, así como de bachilleratos del sistema educativo estatal y federal, entre ellos el Centro de Estudios Tecnológicos Industrial y de Servicios (Cetis) y el Centro de Bachillerato Tecnológico Industrial y de Servicios (Cbtis), al igual que del Colegio de Bachilleres del Estado de Jalisco (Cobaej). Cabe señalar que también ingresan estudiantes de otros municipios del estado, al igual que de otros estados del país.

Para el ingreso se aplica la Prueba de Aptitud Académica del College Board. El puntaje obtenido en esta prueba representa $50 \%$ del puntaje de admisión, el otro $50 \%$ lo aporta el promedio de su certificado de bachillerato.

El ingreso de un estudiante a una licenciatura depende de estos dos factores aunado al cupo de admisión que se establece para cada licenciatura, por lo que se admite a los estudiantes cuya suma de estos dos porcentajes son los mayores hasta completar el cupo establecido en cada ciclo escolar.

Con frecuencia ingresan estudiantes que en alguna área no tienen la adecuada formación académica para cursar de manera satisfactoria sus materias y lograr con ello concluir su licenciatura. Una de ellas es fundamental, el área de matemáticas.

Esta situación no es exclusiva del CUValles, como se puede observar en una gran cantidad de estudios que abordan esta problemática; esta situación se presenta en otras universidades de México y de otros países, por lo que el analizar esta problemática resulta de gran importancia, ya que afecta a alumnos de todo el mundo.

En esa línea, Chen (2016) afirma que un alto porcentaje de estudiantes que ingresa a la universidad llega sin las habilidades académicas necesarias para este nivel educativo, lo que ha conducido a que muchas universidades desarrollen cursos remediales para atender esta situación - aunque, dado que estos cursos cubren contenidos preuniversitarios, los créditos adquiridos no son contabilizados como parte de los requeridos para graduarse-. 


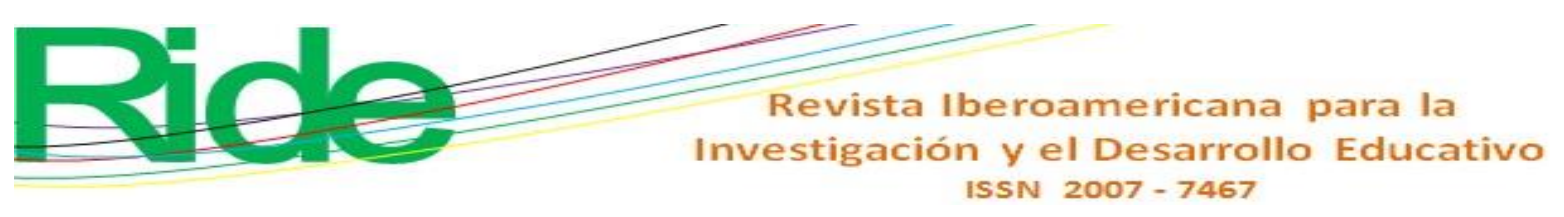

Chen (2016) también subraya que el costo económico asociado a estos cursos remediales es significativo, lo que impacta en los presupuestos públicos.

Sin duda el bachillerato constituye una etapa de formación fundamental para que los aspirantes tengan mayores posibilidades de ingresar a la universidad, al igual que para tener éxito en su trayectoria por la licenciatura de su elección. Al respecto, Herzog (2005) establece que el bachillerato es clave en la retención de alumnos, lo cual es indiscutible no solo por ser el escalón previo a la universidad, sino también por el hecho de que la formación que se desarrolla allí impacta de forma sustancial en el desenvolvimiento durante la etapa universitaria.

En relación con los cursos que suelen presentar dificultades para los estudiantes en las universidades, Adelman (1999, citado en Herzog, 2005) afirma que los tres cursos en los que más reprueban los estudiantes en la universidad son todos del área de matemáticas. De hecho, como resultado de su investigación, Herzog (2005) encontró que el desempeño en esta área es un fuerte indicador de la posible deserción, en específico durante el primer y segundo semestre universitario, de ahí la importancia de atender esta formación desde el bachillerato.

Por su parte, Stone, Alfeld y Pearson (2008), tomando como referencia a Estados Unidos, señalan que los estudiantes de bachillerato no están preparados para las matemáticas que necesitarán en las estaciones posteriores al egreso. También afirman que, contrario a lo que se ha considerado, el incrementar los cursos de esta área no ha sido la solución. Aunado a ello, mencionan que los estudiantes se desenganchan de las matemáticas por su dificultad, la falta de apoyo o simplemente por aburrimiento; el hecho de que muchos de ellos creen que las matemáticas que aprenden en la escuela no son relevantes en su vida es otro de los factores.

Stone (2003) menciona que muchos estudiantes que pretenden ingresar a la universidad requieren cursos remediales ya sea en matemáticas, lectura o escritura, o incluso cursos de las tres disciplinas. Por otra parte, indica que las habilidades matemáticas son básicas para la industria, necesarias no solo para ingenieros y científicos, sino para todos aquellos que quieren avanzar en su camino profesional.

De acuerdo con Anthony (2000, citado en Whannell y Allen, 2012), hay factores que son relevantes en los logros de los estudiantes en cursos introductorios de matemáticas en las universidades: los profesores identifican la cantidad de estudio, falta de esfuerzo y motivación, así como bases matemáticas insuficientes, mientras que los estudiantes 


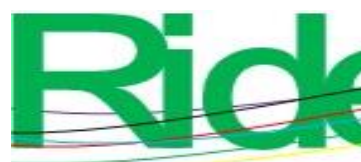

Revista Iberoamericana para la Investigación y el Desarrollo Educativo ISSN $2007-7467$

consideran como factores importantes la enseñanza, el apoyo académico y la pedagogía de los profesores. En los dos casos, se trata de factores que con toda certeza impactan en el desempeño de los alumnos en los cursos iniciales de matemáticas, y en todo aquello que se encuentra enlazado, como es la reprobación y, en no pocos casos, el abandono escolar, por lo que esta situación es muy importante que sea atendida desde diferentes perspectivas.

Moore et al. (2010) mencionan que, en el caso de Texas, uno de los estados más densamente poblados de Estados Unidos, un considerable porcentaje de los egresados de preparatoria carece de una adecuada preparación en lectura y matemáticas para la universidad. En sintonía con lo anterior, Herges, Duffield, Martin y Wageman (2017) comentan que demasiados estudiantes ingresan a la universidad sin el conocimiento y las habilidades matemáticas necesarias para tener éxito y ser competitivos. Los mismos autores consideran como factores que inciden en esta situación la motivación del estudiante, así como la estructura y características de la familia, lo cual influye en los logros en el área de las matemáticas.

Como se ha visto, esta situación no es exclusiva de una universidad o país, dado que suele presentarse en muy variados contextos. Así lo reafirman Long, Iatarola y Conger (2009), quienes enfatizan que un alto porcentaje de los estudiantes de nuevo ingreso de las universidades no están preparados para el nivel requerido en las matemáticas de ese nivel educativo.

Por su parte, Corbishley y Truxaw (2010) encontraron que los profesores perciben que en promedio los estudiantes de nuevo ingreso de las universidades no están preparados en matemáticas. Adicionalmente, especifican en qué aspectos presentan deficiencias: lo más bajos son el dominio de competencias, habilidades algebraicas, razonamiento y generalización.

Ahora bien, hay una aportación importante en relación con deficiencias específicas: Ngo (2019) considera que, en el caso de las habilidades matemáticas básicas, las brechas que comúnmente inhiben el acceso a cursos de nivel superior son la fluidez en los procedimientos con fracciones, la resolución de ecuaciones algebraicas y la respuesta a problemas de palabras. 


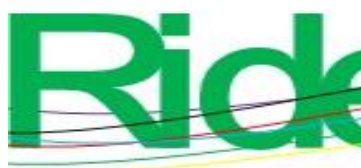

Revista Iberoamericana para la
Investigación y el Desarrollo Educativo
ISSN $2007-7467$

Y retomando a Corbishley y Truxaw (2010), estos investigadores indican que estar preparado para la universidad implica tener habilidades matemáticas específicas. Sugieren que para mejorar la situación es necesario, entre otras acciones, aumentar las expectativas y mejorar la preparación matemática en las etapas anteriores a la universidad.

En el caso de las matemáticas, se suele pensar que incrementando los cursos de matemáticas en el bachillerato ayudará a lograr que los egresados de este nivel educativo logren una mejor formación, sin embargo, Teitelbaum (2003) afirma que este tipo de acciones no mejoran el dominio en esta área.

Problematizando un poco más este punto, Long et al. (2009), al analizar el impacto de tomar cursos avanzados de matemáticas en el bachillerato, observaron que no todos los estudiantes se benefician de ello, y atribuyen las diferencias al rigor y la calidad de los ambientes de aprendizaje, pero, a diferencia de Teitelbaum (2003), consideran que los cursos que los estudiantes toman en el bachillerato contribuyen para que estén preparados para la universidad, en particular señalan el curso de Álgebra 2 como el que tiene mayor impacto, esto en el contexto particular de Estados Unidos.

Siguiendo lo anterior, el incrementar la cantidad de cursos de matemáticas en el bachillerato puede ser una estrategia que considerar para su aplicación en el contexto de los bachilleratos de la región de los Valles, la cual es la principal zona de influencia del CUValles.

A pesar de las diferencias culturales, económicas y sociales de los países, hay coincidencia en la problemática presente entre los estudiantes que egresan de bachillerato y entran a la universidad, en el caso concreto, las deficiencias académicas en la formación en las matemáticas.

Parte de la importancia de evaluar cómo llegan en la formación matemática los estudiantes del bachillerato a las universidades, y en específico al CUValles, tiene que ver con lo que expone Camara (2013), cuya investigación muestra que las mediciones cognitivas de desempeño académico, como lo son las calificaciones del bachillerato y los resultados de pruebas, son altamente predictivas de las calificaciones obtenidas en la universidad. Cabe señalar que, en no pocos casos, los estudiantes que ingresan al CUValles presentan un promedio de calificaciones del bachillerato que no necesariamente coincide con el puntaje de la prueba de aptitud académica que presentan para su ingreso, incluidos los puntajes en el área de matemáticas. 


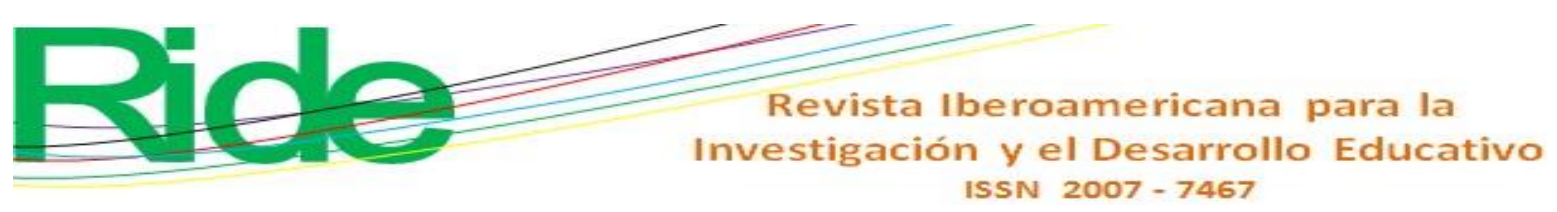

\section{Objetivo}

El objetivo de esta investigación es identificar los conocimientos básicos en matemáticas de los estudiantes de primer ingreso de las licenciaturas de Administración, Turismo, Contaduría Pública, Tecnologías de la Información y las ingenierías en Mecatrónica, Electrónica y Computación, Instrumentación Electrónica y Nanosensores, Diseño Molecular de Materiales, Geofísica y Sistemas Biológicos. Esto para fortalecer las deficiencias encontradas y así estas no sean factor de reprobación, rezago y, en el peor de los casos, de abandono de los estudios de licenciatura.

\section{Metodología}

La información se obtuvo mediante la aplicación de un examen en el que se evaluaron conocimientos matemáticos básicos, los cuales se consideraron con base en los programas de estudios de nivel medio superior. Se determinó como universo de estudio al total de estudiantes de primer ingreso de la licenciatura en Tecnologías de la información, ingeniería en Mecatrónica, ingeniería en Electrónica y Computación, ingeniería en Instrumentación Electrónica y Nanosensores, ingeniería en Diseño Molecular de Materiales, ingeniería en Geofísica, ingeniería en Sistemas Biológicos, licenciatura en Administración, licenciatura en Turismo y licenciatura en Contaduría Pública del CUValles de la Universidad de Guadalajara del ciclo 2019B (agosto 2019-enero 2020). La prueba se aplicó en la primera sesión de cada uno de los grupos y solo se permitió lápiz y borrador.

El examen que se aplicó constó de 15 reactivos de opción múltiple (ver anexo). En conjunto, los reactivos demandaban conocimientos básicos de operaciones aritméticas tales como la suma y multiplicación de fracciones, la jerarquía de las operaciones, leyes de los signos y de los exponentes, desarrollo del cuadrado de un binomio, traducción de lenguaje natural a lenguaje matemático, traducción de información geométrica a lenguaje algebraico y solución de ecuaciones lineal y cuadrática.

El análisis estadístico se realizó utilizando el programa Excel y Minitab. En la tabla 1 se resume la conformación de la población. Se analizaron las respuestas de los estudiantes de primer ingreso de 10 licenciaturas del CUValles. 
Tabla 1. Distribución de la población por programa educativo

\begin{tabular}{|l|c|}
\hline Programa educativo & Total de alumnos \\
\hline Licenciatura en Tecnologías de la información & 54 \\
\hline Ingeniería en Mecatrónica & 34 \\
\hline Ingeniería en Electrónica y Computación & 33 \\
\hline $\begin{array}{l}\text { Ingeniería en Instrumentación Electrónica y } \\
\text { Nanosensores }\end{array}$ & 20 \\
\hline Ingeniería en Diseño Molecular de Materiales & 35 \\
\hline Ingeniería en Geofísica & 72 \\
\hline Ingeniería en Sistemas Biológicos & 36 \\
\hline Licenciatura en Administración & 80 \\
\hline Licenciatura en Turismo & 413 \\
\hline Licenciatura en Contaduría Pública & \\
\hline Total & \\
\hline
\end{tabular}

Fuente: Elaboración propia

\section{Resultados}

Gracias al análisis de la información obtenida fue posible identificar el número de respuestas correctas, incorrectas y sin contestar del total de estudiantes, lo cual se puede observar en la tabla 2.

Tabla 2. Distribución de las respuestas para el total de estudiantes.

\begin{tabular}{|l|r|}
\hline Tipo de respuestas & Frecuencia \\
\hline Correctas & 3282 \\
\hline Incorrectas & 2738 \\
\hline Sin contestar & 175 \\
\hline
\end{tabular}

Fuente: Elaboración propia

Asimismo, en la figura 1 se observa que $52.98 \%$ de las respuestas fueron correctas, mientras que $44.20 \%$ fueron incorrectas. 


\section{Revista Iberoamericana para la Investigación y el Desarrollo Educativo ISSN $2007-7467$}

Tabla 3. Total de estudiantes que obtuvieron cantidad de aciertos

\begin{tabular}{|c|c|c|}
\hline $\begin{array}{l}\text { Cantidad de } \\
\text { aciertos }\end{array}$ & $\begin{array}{l}\text { Cantidad de } \\
\text { estudiantes }\end{array}$ & Porcentaje \\
\hline 1 & 0 & 0 \\
\hline 2 & 1 & 0.26 \\
\hline 3 & 8 & 2.10 \\
\hline 3 & 12 & 3.15 \\
\hline 4 & 24 & 6.31 \\
\hline 5 & 45 & 11.84 \\
\hline 6 & 49 & 12.89 \\
\hline 7 & 56 & 14.73 \\
\hline 8 & 45 & 11.84 \\
\hline 9 & 45 & 11.84 \\
\hline 10 & 50 & 13.15 \\
\hline 11 & 28 & 7.36 \\
\hline 12 & 20 & 5.26 \\
\hline 13 & 14 & 3.68 \\
\hline 14 & 13 & 3.42 \\
\hline 15 & 3 & 0.78 \\
\hline
\end{tabular}

Fuente: Elaboración propia 


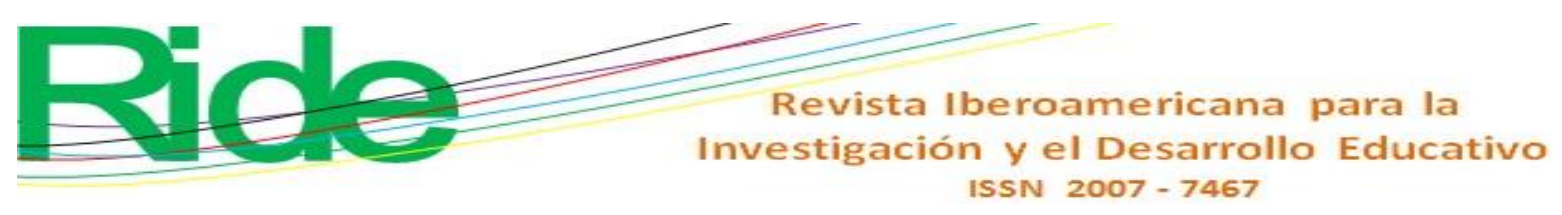

Figura 2. Total de estudiantes que obtuvieron cantidad de aciertos

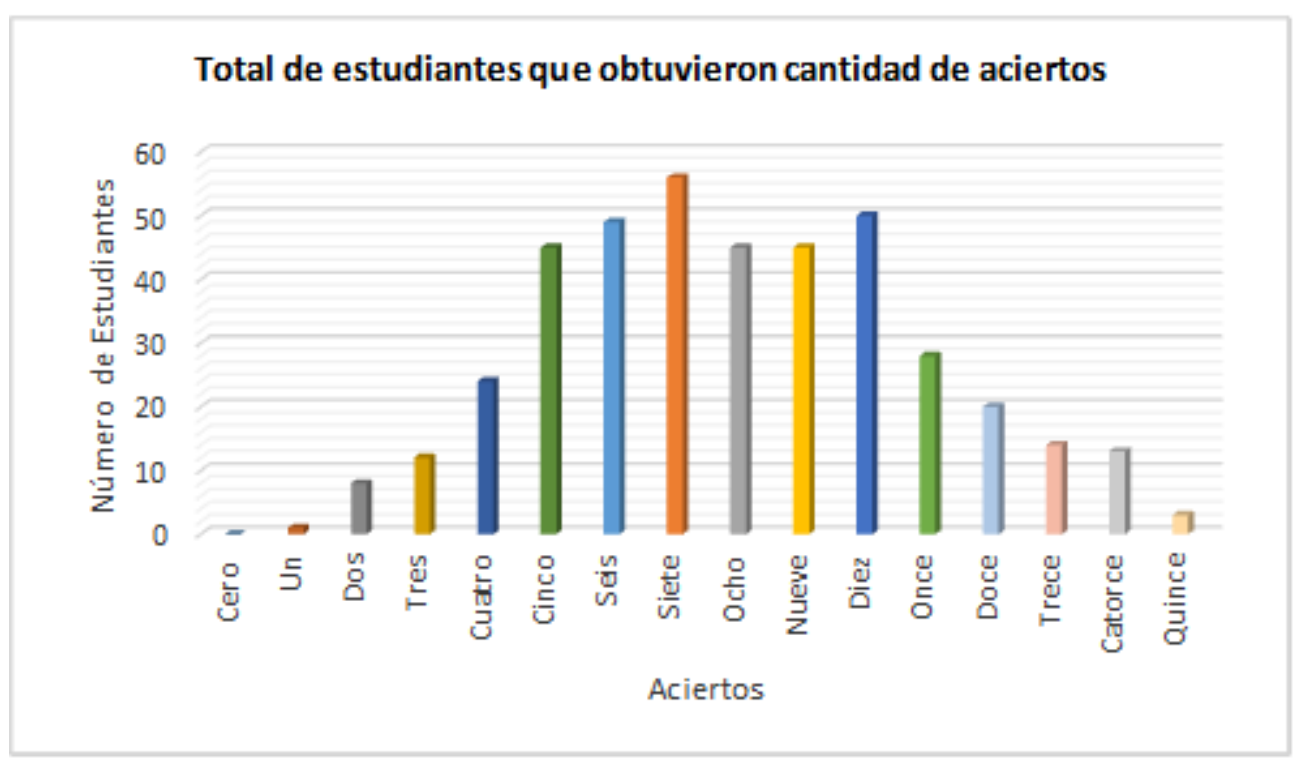

Fuente: Elaboración propia

En la misma figura 3, como en la tabla 4, se observan los reactivos con más respuestas incorrectas: el número 13 con $11.562 \%$, el número cinco con $10.719 \%$, el número 10 con $10.558 \%$ y el número 15 con $10.157 \%$. En el reactivo número 13 se requiere expresar algebraicamente el perímetro de un rectángulo a partir de una expresión verbal; el reactivo cinco consiste en simplificar una expresión aplicando los conceptos de término semejante, leyes de los signos y uso de paréntesis; el reactivo 10 solicita identificar el desarrollo de un binomio al cuadrado, y el reactivo 15 requiere resolver una ecuación cuadrática. Teniendo en cuenta lo anterior, se coincide con lo expresado por Long et al. (2009) cuando afirman que un alto porcentaje de los estudiantes de nuevo ingreso de las universidades no está preparado para el nivel requerido en las matemáticas.

También podemos ver y es de llamar la atención que el reactivo número 15 es el que más estudiantes no contestaron ( 28 estudiantes no lo contestaron, lo que representa $18.79 \%$ de la población). 
Tabla 4. Total de estudiantes por tipo de respuesta

\begin{tabular}{|c|c|c|c|c|c|c|}
\hline \multirow[t]{2}{*}{ Reactivo } & \multicolumn{2}{|c|}{ Correcta } & \multicolumn{2}{|c|}{ Incorrecta } & \multicolumn{2}{|c|}{ Sin contestar } \\
\hline & Frecuencia & $\%$ & Frecuencia & $\%$ & Frecuencia & $\%$ \\
\hline 1 & 206 & 6.73 & 204 & 8.18 & 2 & 1.34 \\
\hline 2 & 195 & 6.37 & 213 & 8.55 & 5 & 3.35 \\
\hline 3 & 326 & 10.65 & 78 & 3.13 & 9 & 6.04 \\
\hline 4 & 273 & 8.92 & 129 & 5.17 & 11 & 7.38 \\
\hline 5 & 124 & 4.05 & 267 & 10.71 & 22 & 14.76 \\
\hline 6 & 155 & 5.06 & 235 & 9.43 & 23 & 15.43 \\
\hline 7 & 291 & 9.51 & 117 & 4.69 & 6 & 4.02 \\
\hline 8 & 269 & 8.79 & 136 & 5.45 & 8 & 5.36 \\
\hline 9 & 197 & 6.43 & 205 & 8.22 & 11 & 7.38 \\
\hline 10 & 140 & 4.57 & 263 & 10.55 & 10 & 6.71 \\
\hline 11 & 215 & 7.02 & 190 & 7.62 & 8 & 5.36 \\
\hline 12 & 324 & 10.59 & 80 & 3.21 & 9 & 6.04 \\
\hline 13 & 111 & 3.62 & 288 & 11.56 & 15 & 10.06 \\
\hline 14 & 327 & 10.69 & 80 & 3.21 & 8 & 5.36 \\
\hline 15 & 129 & 4.21 & 253 & 10.15 & 28 & 18.79 \\
\hline
\end{tabular}

Fuente: Elaboración propia 


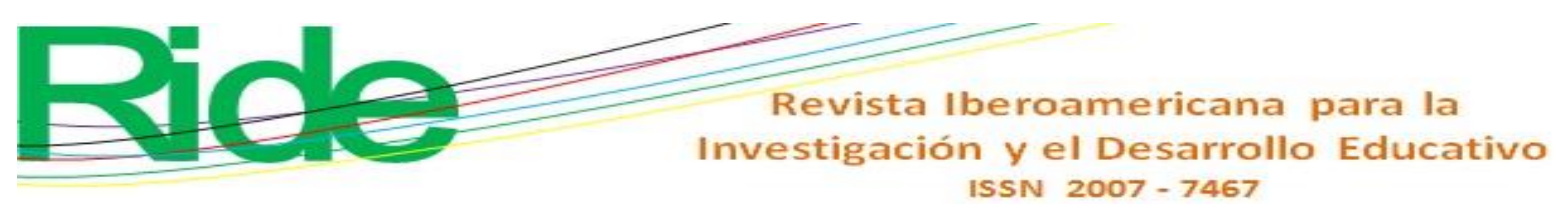

Figura 3. Tipo de respuesta por reactivo correctamente, incorrectamente y sin contestar

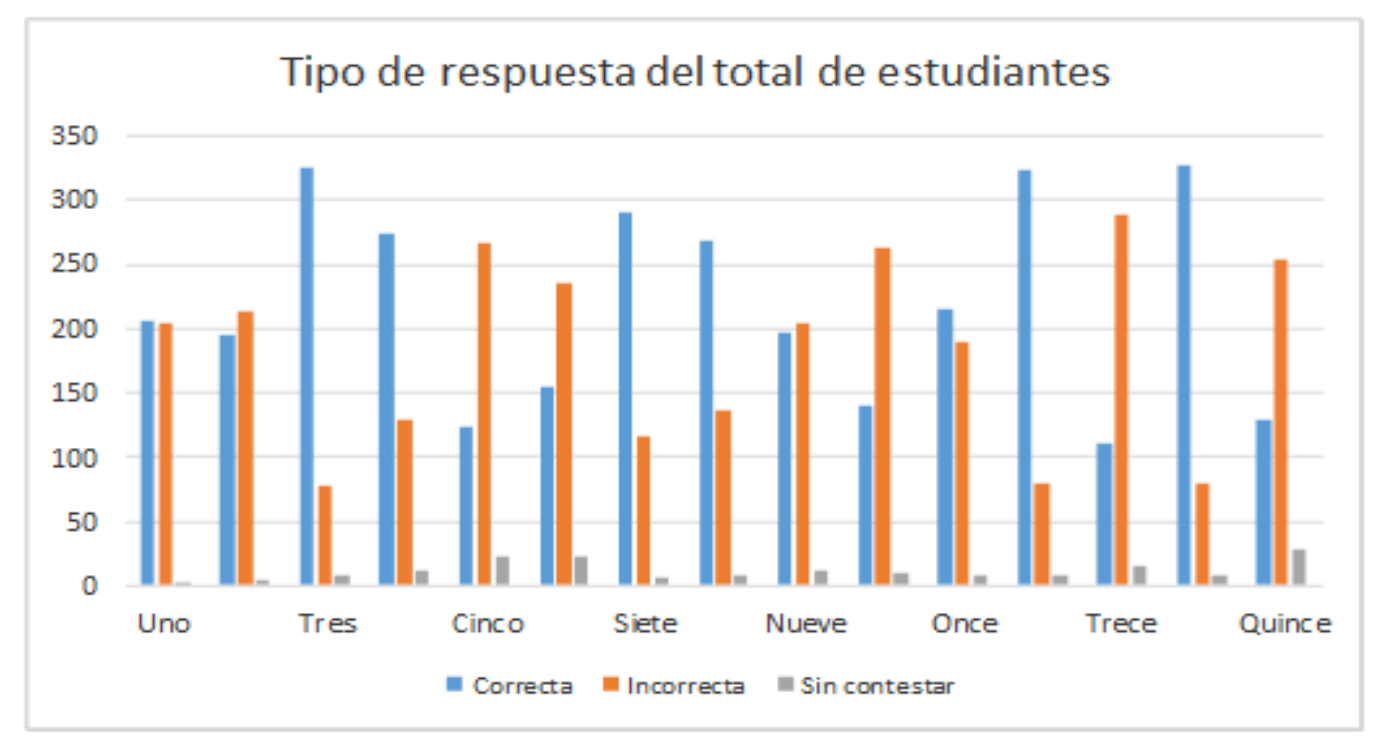

Fuente: Elaboración propia

Por otra parte, en la tabla 5 es posible apreciar que el promedio general obtenido fue de 52.978, con una desviación de 19.316. Este valor nos indica qué tan alejados están los datos con respecto a la media, es decir, es un indicador de que los datos oscilan a esta distancia de la media (Gutiérrez y Vladimirovna, 2017; Levin y Rubin, 2004). Dicho resultado coincide con el obtenido por Gamboa, Castillo y Hidalgo (2019), cuyos participantes en un estudio similar obtuvieron una nota no aprobatoria.

Tabla 5. Promedio y desviación estándar del total de estudiantes

\begin{tabular}{|l|c|}
\hline Promedio general & 52.978 \\
\hline Desviación estándar & 19.316 \\
\hline
\end{tabular}

Fuente: Elaboración propia

La tabla 6 muestra el promedio obtenido por género: el promedio obtenido por las mujeres fue 50.292 y el de los hombres fue de 54.876 .

Tabla 6. Promedio por género del total de estudiantes

\begin{tabular}{|l|r|r|c|}
\hline Género & \multicolumn{1}{|c|}{ Promedio } & \multicolumn{1}{|c|}{ Total } & Desviación estándar \\
\hline Hombres & 54.876 & 242 & 19.391 \\
\hline Mujeres & 50.292 & 171 & 18.942 \\
\hline & & 413 & \\
\hline
\end{tabular}

Fuente: Elaboración propia 


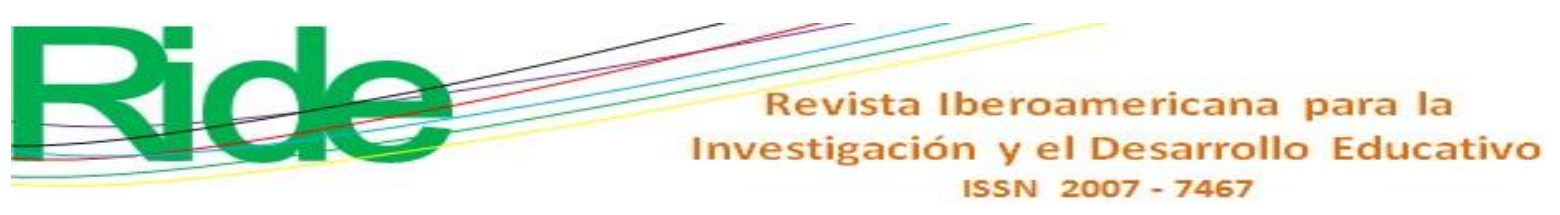

En relación con el porcentaje de respuestas correctas e incorrectas, al realizar la comparación entre los programas educativo se encontró que los estudiantes de ingeniería en Mecatrónica obtuvieron el mayor porcentaje de respuestas correctas con $68.64 \%$, seguidos por los estudiantes de ingeniería en Geofísica con $62.74 \%$ y los de ingeniería en Diseño Molecular de Materiales con $58 \%$. Por otra parte, los estudiantes de la licenciatura en Turismo obtuvieron el mayor porcentaje de respuestas incorrectas con $58.51 \%$, seguidos por los de ingeniería en Sistemas Biológicos con 54.09 \% y los de la licenciatura en Tecnologías de la Información con $49.79 \%$. En lo correspondiente al número de respuestas sin contestar, el mayor porcentaje lo registró la ingeniería en Diseño Molecular de Materiales con 7.33 \%, como se observa en la tabla 7. Además, en la figura 4 se puede advertir la comparación entre las respuestas correctas e incorrectas de los estudiantes entre los programas educativos. 
Tabla 7. Número de respuestas y porcentajes por programa educativo

\begin{tabular}{|c|c|c|c|c|c|c|}
\hline & \multicolumn{5}{|c|}{ Tipo de respuestas } & \multirow[b]{2}{*}{$\%$} \\
\hline $\begin{array}{l}\text { Programa } \\
\text { educativo }\end{array}$ & Correctas & $\%$ & Incorrectas & $\%$ & $\begin{array}{l}\text { Sin } \\
\text { contestar }\end{array}$ & \\
\hline $\begin{array}{l}\text { Licenciatura en } \\
\text { Tecnologías de } \\
\text { la información }\end{array}$ & 235 & $\begin{array}{c}48.9 \\
5\end{array}$ & 239 & $\begin{array}{c}49.7 \\
9\end{array}$ & 6 & $\begin{array}{c}1.2 \\
5\end{array}$ \\
\hline $\begin{array}{l}\text { Ingeniería en } \\
\text { Mecatrónica }\end{array}$ & 556 & $\begin{array}{c}68.6 \\
4\end{array}$ & 237 & $\begin{array}{c}29.2 \\
5\end{array}$ & 17 & $\begin{array}{c}2.0 \\
9\end{array}$ \\
\hline $\begin{array}{l}\text { Ingeniería en } \\
\text { Electrónica } \quad \text { y } \\
\text { Computación }\end{array}$ & 271 & $\begin{array}{c}53.1 \\
3\end{array}$ & 216 & $\begin{array}{c}42.3 \\
5\end{array}$ & 23 & $\begin{array}{c}4.5 \\
0\end{array}$ \\
\hline $\begin{array}{l}\text { Ingeniería en } \\
\text { Instrumentació } \\
\text { n Electrónica y } \\
\text { Nanosensores }\end{array}$ & 268 & $\begin{array}{c}54.1 \\
4\end{array}$ & 218 & $\begin{array}{c}44.0 \\
4\end{array}$ & 9 & $\begin{array}{c}1.8 \\
1\end{array}$ \\
\hline $\begin{array}{ll}\text { Ingeniería } & \text { en } \\
\text { Diseño } & \\
\text { Molecular } & \text { de } \\
\text { Materiales } & \end{array}$ & 174 & 58 & 104 & $\begin{array}{c}34.6 \\
6\end{array}$ & 22 & $\begin{array}{c}7.3 \\
3\end{array}$ \\
\hline $\begin{array}{l}\text { Ingeniería en } \\
\text { Geofísica }\end{array}$ & 160 & $\begin{array}{c}62.7 \\
4\end{array}$ & 88 & $\begin{array}{c}34.5 \\
0\end{array}$ & 7 & $\begin{array}{c}2.7 \\
4\end{array}$ \\
\hline $\begin{array}{ll}\text { Ingeniería en } \\
\text { Sistemas } \\
\text { Biológicos }\end{array}$ & 232 & $\begin{array}{c}44.1 \\
9\end{array}$ & 284 & $\begin{array}{c}54.0 \\
9\end{array}$ & 9 & $\begin{array}{c}1.7 \\
1\end{array}$ \\
\hline $\begin{array}{l}\text { Licenciatura en } \\
\text { Administración }\end{array}$ & 528 & $\begin{array}{c}48.8 \\
8\end{array}$ & 511 & $\begin{array}{c}47.3 \\
1\end{array}$ & 41 & $\begin{array}{c}3.7 \\
9\end{array}$ \\
\hline $\begin{array}{l}\text { Licenciatura en } \\
\text { Turismo }\end{array}$ & 204 & $\begin{array}{c}37.7 \\
7\end{array}$ & 316 & $\begin{array}{c}58.5 \\
1\end{array}$ & 20 & $\begin{array}{c}3.7 \\
0\end{array}$ \\
\hline $\begin{array}{l}\text { Licenciatura en } \\
\text { Contaduría } \\
\text { Pública }\end{array}$ & 654 & 54.5 & 525 & $\begin{array}{c}43.7 \\
5\end{array}$ & 21 & $\begin{array}{c}1.7 \\
5\end{array}$ \\
\hline
\end{tabular}

Fuente: Elaboración propia 
Tabla 8. Porcentaje de respuestas correctas por reactivo y por programa educativo

\begin{tabular}{|c|c|c|c|c|c|c|c|c|c|c|}
\hline & 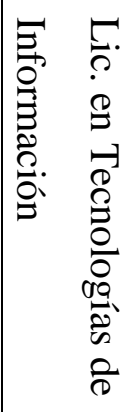 & 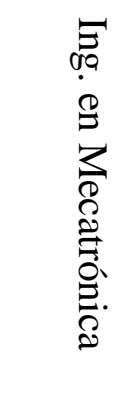 & 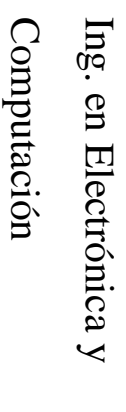 & 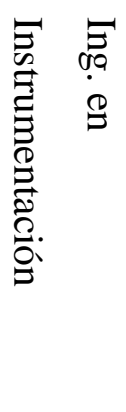 & 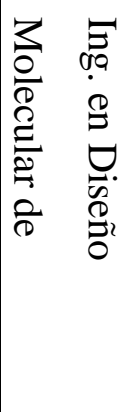 & 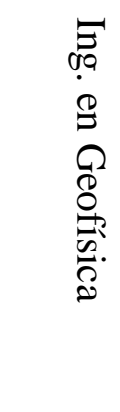 & 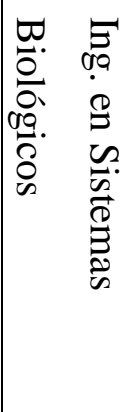 & 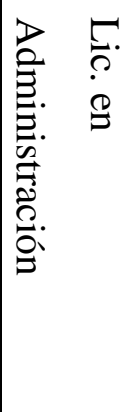 & $\begin{array}{l}5 . \\
\hat{0} \\
8 \\
\Xi \\
\Xi \\
\Xi \\
气 \\
0\end{array}$ & 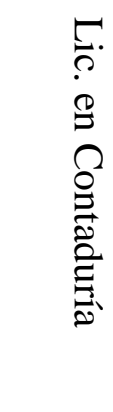 \\
\hline R1 & 25 & 68.51 & 41.17 & 63.63 & 45 & 88.23 & 45.71 & 50 & 30.55 & 48.75 \\
\hline $\mathrm{R} 2$ & 25 & 79.62 & 38.23 & 54.54 & 65 & 94.11 & 28.57 & 34.72 & 16.66 & 53.75 \\
\hline R3 & $\begin{array}{c}59.37 \\
5\end{array}$ & 96.29 & 88.23 & 93.93 & 85 & 70.58 & 85.71 & 75 & 58.33 & 75 \\
\hline $\mathrm{R} 4$ & $\begin{array}{c}71.87 \\
5\end{array}$ & 94.44 & 58.82 & 78.78 & 65 & 64.70 & 45.71 & 63.88 & 41.66 & 65 \\
\hline R5 & $\begin{array}{c}46.87 \\
5\end{array}$ & 33.33 & 38.23 & 18.18 & 5 & 58.82 & 20 & 30.55 & 22.22 & 30 \\
\hline R6 & $\begin{array}{c}34.37 \\
5\end{array}$ & 51.85 & 41.17 & 9.09 & 45 & 29.41 & 48.57 & 37.5 & 25 & 40 \\
\hline R7 & 65.62 & 72.22 & 61.76 & 69.69 & 70 & 82.35 & 74.28 & 75 & 72.22 & 66.25 \\
\hline $\mathrm{R} 8$ & 46.87 & 75.92 & 73.52 & 72.72 & 75 & 64.70 & 54.28 & 58.33 & 55.55 & 71.25 \\
\hline R9 & 43.75 & 64.81 & 58.82 & 57.57 & 65 & 70.58 & 28.57 & 37.5 & 25 & 47.5 \\
\hline R10 & $\begin{array}{c}34.37 \\
5\end{array}$ & 59.25 & 23.52 & 36.36 & 25 & 76.47 & 20 & 25 & 19.44 & 33.75 \\
\hline R11 & 56.25 & 61.11 & 58.82 & 60.60 & 70 & 35.29 & 40 & 51.38 & 41.66 & 47.5 \\
\hline R12 & 84.37 & 83.33 & 94.11 & 69.69 & 95 & 64.70 & 74.28 & 69.44 & 58.33 & 87.5 \\
\hline R13 & 34.37 & 46.29 & 20.58 & 27.27 & 45 & 11.76 & 11.42 & 18.05 & 19.44 & 30 \\
\hline R14 & 81.25 & 96.29 & 85.29 & 69.69 & 75 & 76.47 & 65.71 & 77.77 & 63.88 & 83.75 \\
\hline R15 & 25 & 46.29 & 14.70 & 30.30 & 40 & 52.94 & 20 & 29.16 & 16.66 & 37.5 \\
\hline
\end{tabular}

Fuente: Elaboración propia

La tabla 8 muestra el porcentaje de las respuestas correctas que registró cada programa educativo en cada uno de los reactivos. Es posible observar que ingeniería en Mecatrónica tiene el mayor porcentaje de aciertos correctos en los reactivos tres (jerarquía de operaciones), cuatro (propiedad distributiva y ley de signos), seis (propiedad distributiva 


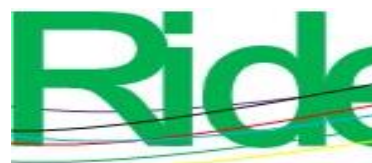

Revista Iberoamericana para la
Investigación y el Desarrollo Educativo
ISSN $2007-7467$

de la división), ocho (multiplicación de monomios con una misma base y exponente diferente), 13 (traducción de lenguaje natural a matemático) y 14 (solución de una ecuación lineal en una variable); ingeniería en Geofísica tiene mayor porcentaje de aciertos correctos en los reactivos uno (multiplicación de fracciones), dos (división de fracciones), cinco (signos y exponentes), siete (ley de los exponentes), nueve (división de monomios con una misma base), 10 (binomio al cuadrado) y 15 (solución ecuación cuadrática); el programa educativo de ingeniería en Diseño Molecular de Materiales tiene mayor porcentaje en los reactivos 12 (traducir información visual a lenguaje matemático) y tres (jerarquía de operaciones). También se puede observar que el reactivo cinco (signos y exponentes) tiene el menor porcentaje, seguido del reactivo seis (propiedad distributiva de la división), en el programa educativo de ingeniería en Instrumentación Electrónica y Nanosensores. Además, el reactivo 13 (traducción de lenguaje natural a matemático), en el programa educativo de ingeniería en Geofísica, tiene un bajo porcentaje de respuestas correctas. Aunado a ello, el reactivo 15 (solución ecuación cuadrática) también tiene un bajo porcentaje de aciertos por parte de los participantes de la ingeniería en Electrónica y Computación. Aquí se coincide con lo que señala Ngo (2019) al afirmar que las brechas de habilidades que comúnmente inhiben el acceso a cursos de nivel superior son la fluidez en los procedimientos con fracciones, la resolución de ecuaciones algebraicas y la respuesta a problemas de palabras.

En la figura 5 se pueden observar las respuestas correctas de los reactivos uno, dos y tres por programa educativo. En el reactivo uno, el mayor porcentaje de respuestas correctas lo obtuvieron los estudiantes de la ingeniería en Geofísica y el menor los de la licenciatura en Tecnologías de la Información. En el reactivo dos, el mayor porcentaje de respuestas correctas lo obtuvieron también los estudiantes de ingeniería en Geofísica y el menor los de la licenciatura en Turismo. Por último, en cuanto al reactivo tres, el mayor porcentaje de respuestas correctas lo obtuvieron los estudiantes de ingeniería en Mecatrónica y el menor los de la licenciatura en Turismo. 


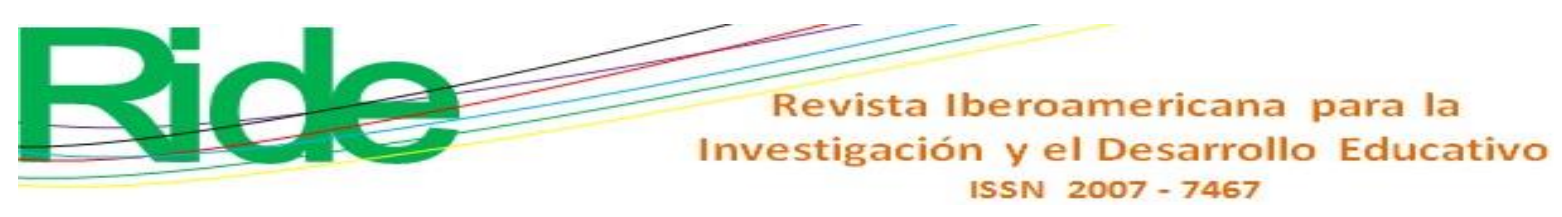

Figura 5. Respuestas correctas en los reactivos uno, dos, tres por programa educativo

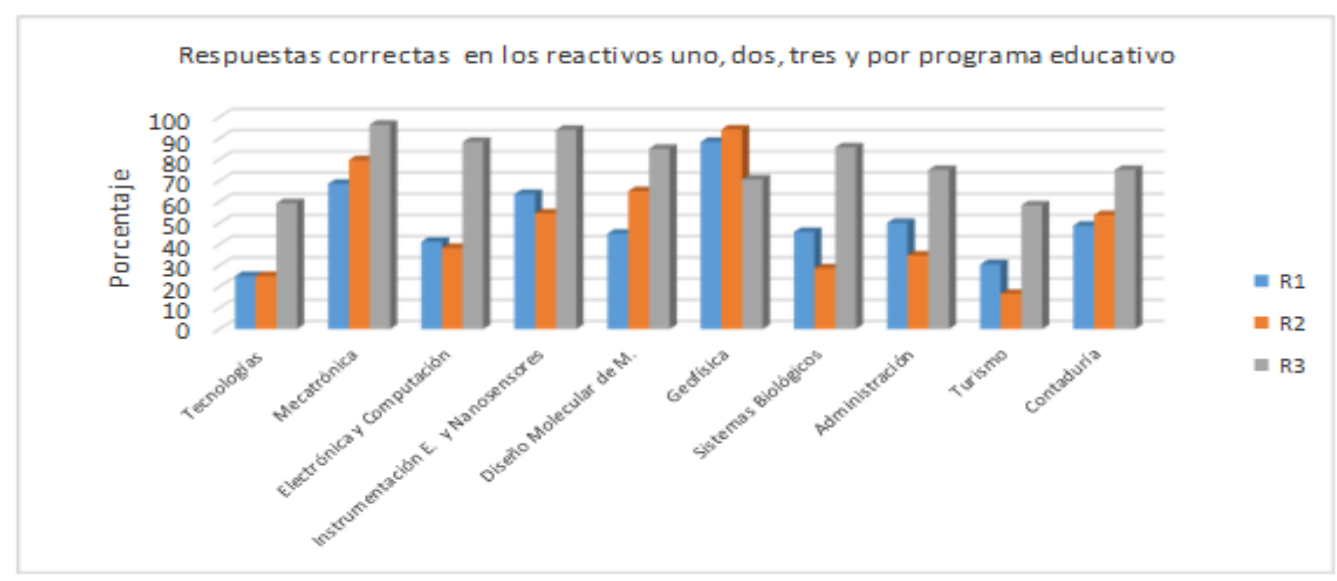

Fuente: Elaboración propia

En la figura 6 se observa que casi todos los estudiantes del programa educativo de ingeniería en Mecatrónica, $94.4 \%$, contestaron el reactivo cuatro correctamente. La ingeniería en Diseño Molecular de Materiales registró que el reactivo que menos contestaron correctamente fue el cinco, con $5 \%$. Cabe subrayar que este reactivo es el que más se les complicó a los estudiantes de los diferentes programas educativos, a excepción de los de ingeniería en Geofísica e ingeniería en Instrumentación Electrónica y Nanosensores.

Figura 6. Respuestas correctas en $\operatorname{los}$ reactivos 4, 5, 6 y 7 por programa educativo

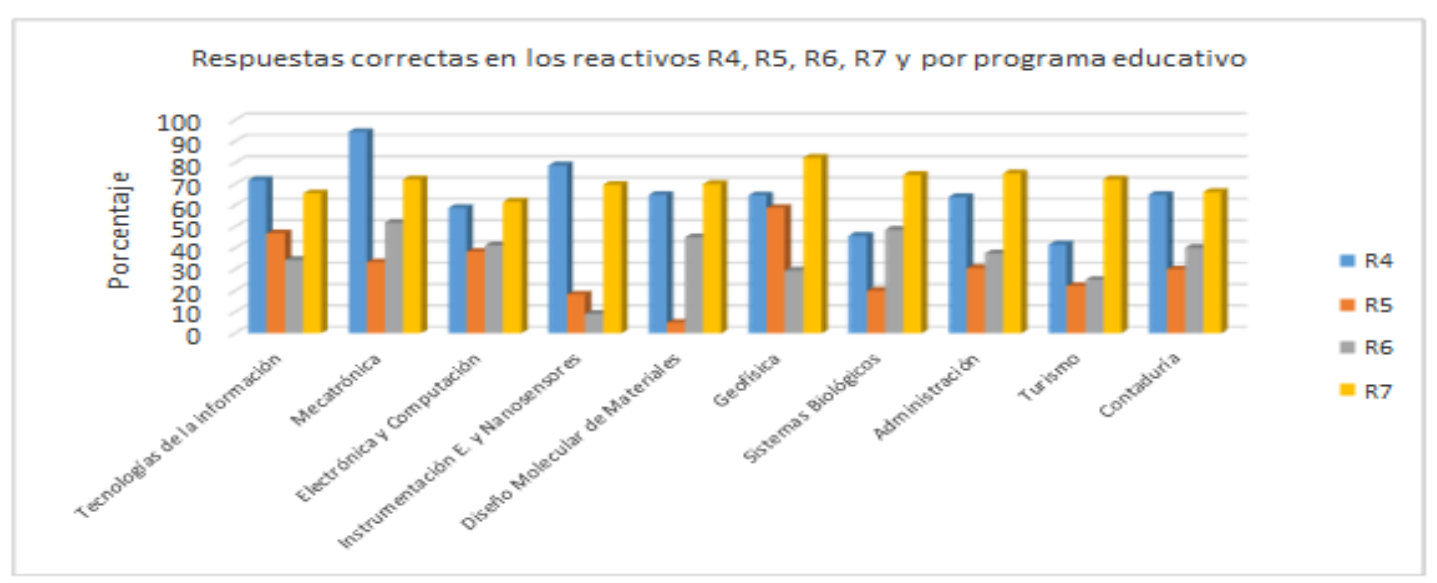

Fuente: Elaboración propia 


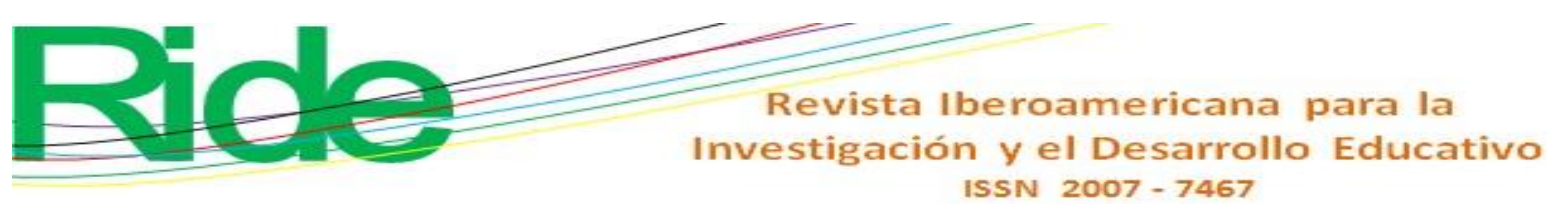

Figura 7. Respuestas correctas en los reactivos $8,9,10$ y 11 por programa educativo

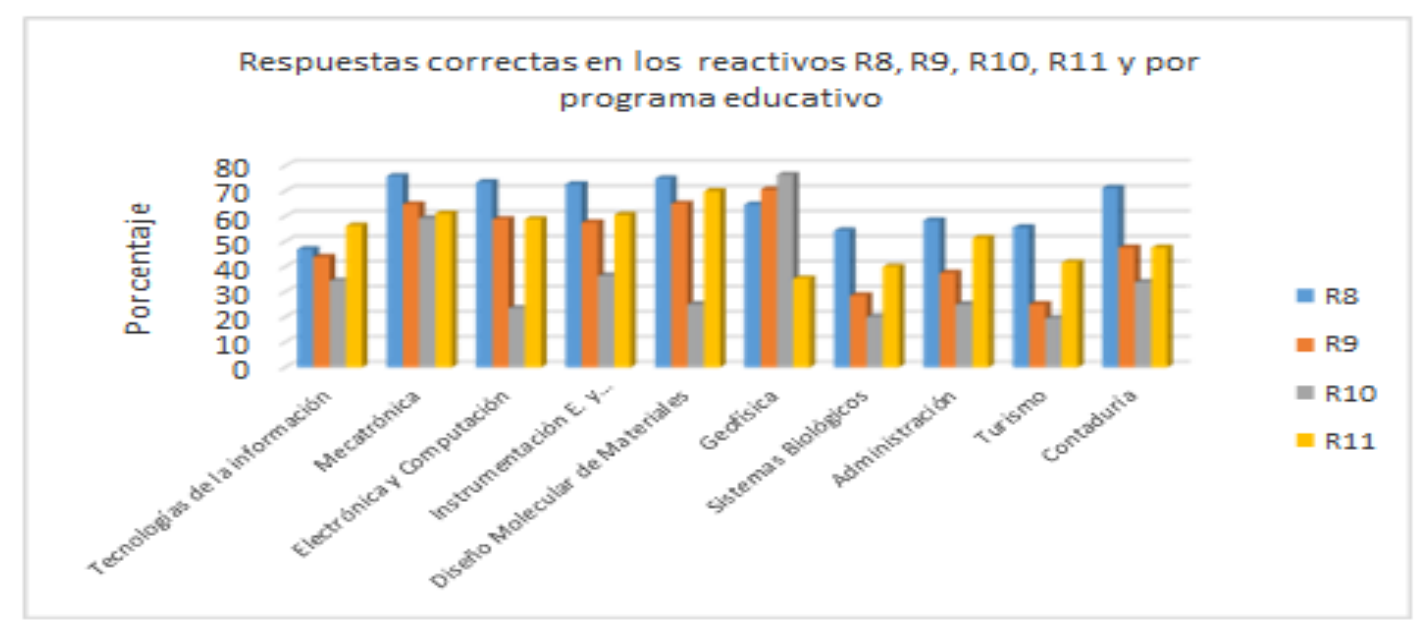

Fuente: Elaboración propia

En la figura 7 se puede observar, por su parte, que los estudiantes del programa de ingeniería en Mecatrónica son quienes obtuvieron el mayor porcentaje de respuestas correctas en el reactivo ocho, mientras que en el caso del reactivo nueve el mayor porcentaje lo obtuvo la ingeniería en Geofísica. Por otra parte, se observa que en el reactivo 10 todos los programas educativos tuvieron un bajo porcentaje de respuestas correctas, excepto la ingeniería en Geofísica.

Finalmente, en la gráfica de la figura 8 se observa que los reactivos 12 y 14 son los que obtuvieron mayor porcentaje de respuestas correctas en todos los programas educativos, mientras que en el 13 y 15 los porcentajes fueron bajos para todos los programas educativos.

Figura 8. Respuestas correctas en los reactivos 12, 13, 14 y 15 por programa educativo

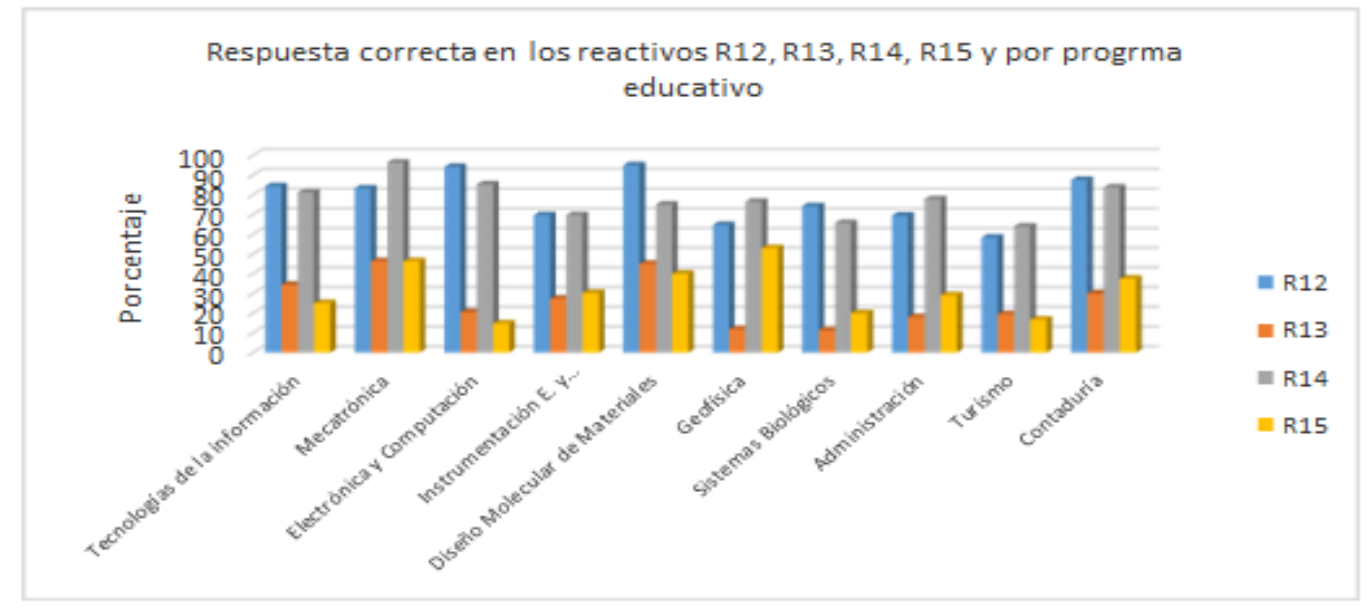

Fuente: Elaboración propia 


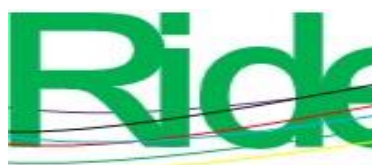

Revista Iberoamericana para la
Investigación y el Desarrollo Educativo
ISSN $2007-7467$

Como queda patente en los resultados descritos previamente, los estudiantes presentan deficiencias en conocimientos básicos de matemáticas, situación similar a la que encontraron Gamboa et al. (2019).

Figura 9. Promedio por programa educativo

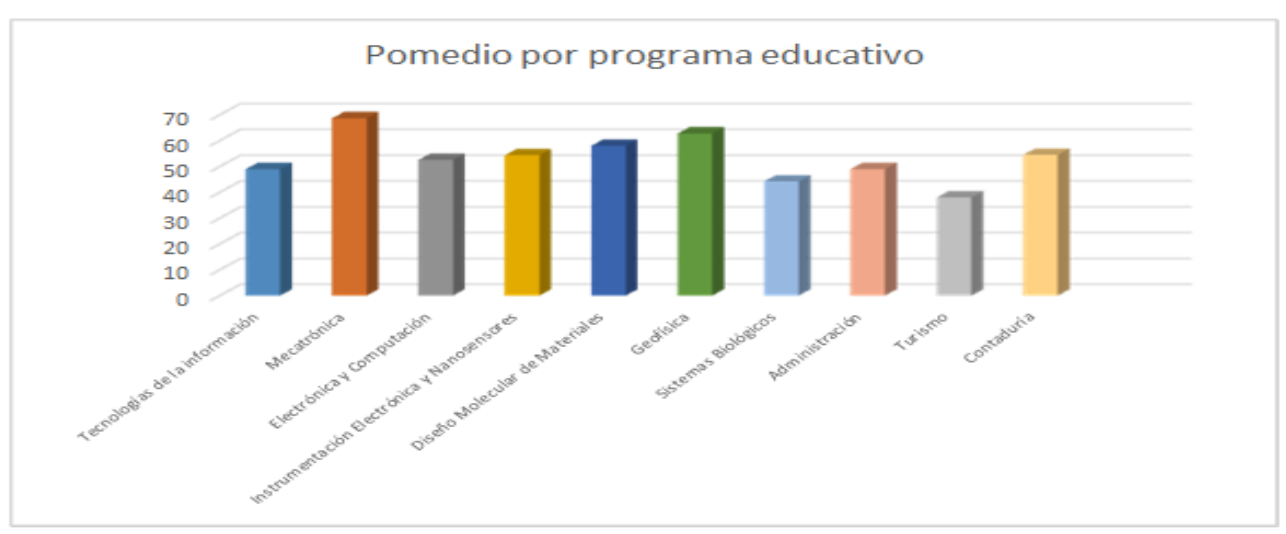

Fuente: Elaboración propia

Ahora bien, la figura 9 muestra el promedio de calificaciones de los alumnos de los diversos programas educativos. Allí se puede observar que el mayor promedio lo obtuvieron los estudiantes de ingeniería en Mecatrónica y el menor los de la licenciatura en Turismo. Con respecto a esto, resalta que la mayoría de los promedios no son aprobatorios, por lo que se coincide con lo externado por Corbishley y Truxaw (2010), quienes afirman que los profesores perciben que en promedio los estudiantes de nuevo ingreso de las universidades no están preparados en matemáticas.

La tabla 9 presenta el promedio y la desviación estándar por programa educativo. Se observa que el promedio mayor lo tiene el programa educativo de ingeniería en Mecatrónica, con una desviación estándar de 18.389 (esto significa que todos los datos oscilan entre un rango de esta cantidad por arriba de la media y esta cantidad por debajo de la media); le sigue el programa educativo de ingeniería en Geofísica, con un promedio de 62.745, pero su desviación estándar es mayor que en ingeniería en Mecatrónica. El programa educativo con menor promedio es la licenciatura en Turismo, también con una desviación estándar menor que todos los programas educativos, lo cual significa que la gran mayoría está alrededor de ese promedio.

Un diagrama de caja muestra la dispersión o separación de los datos. La dispersión puede medirse por la longitud o rango del diagrama, ya que desde el mínimo hasta el valor máximo estaría la totalidad de los datos, por lo que dentro de la caja estaría representado 


\section{Revista Iberoamericana para la Investigación y el Desarrollo Educativo ISSN $2007-7467$}

$50 \%$ de los datos (del primer cuartil al segundo cuartil está $25 \%$ de los datos, el segundo cuartil es la mediana y de este al tercer cuartil está otro $25 \%$ de los datos); la dispersión dentro de la caja se mide por el rango intercuartil, es decir, el tercer cuartil menos el primer cuartil expresará la variabilidad de 50 \% de los datos (Gutiérrez y Vladimirovna, 2017; Levin y Rubin, 2004).

Tabla 9. Promedio de los estudiantes por programa educativo

\begin{tabular}{|l|r|c|}
\hline \multicolumn{1}{|c|}{ Programa educativo } & Promedio & $\begin{array}{r}\text { Desviación } \\
\text { estándar }\end{array}$ \\
\hline $\begin{array}{l}\text { Licenciatura en Tecnologías de la } \\
\text { información }\end{array}$ & 48.958 & 16.984 \\
\hline Ingeniería Mecatrónica & 68.641 & 18.389 \\
\hline Ingeniería en Electrónica y Computación & 52.549 & 16.637 \\
\hline $\begin{array}{l}\text { Ingeniería en Instrumentación Electrónica y } \\
\text { Nanosensores }\end{array}$ & 54.343 & 21.023 \\
\hline $\begin{array}{l}\text { Ingeniería en Diseño Molecular de } \\
\text { Materiales }\end{array}$ & 58 & 20.869 \\
\hline Ingeniería en Geofísica & 44.19 & 16.887 \\
\hline Ingeniería en Sistemas Biológicos & 48.981 & 17.121 \\
\hline Licenciatura en Administración & 37.96 & 16.237 \\
\hline Licenciatura en Turismo & 54.5 & 16.356 \\
\hline Licenciatura en Contaduría & & 19.868 \\
\hline
\end{tabular}

Fuente: Elaboración propia 


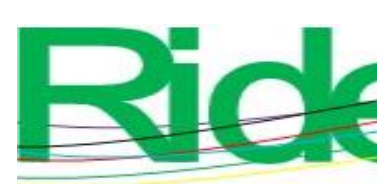

Revista Iberoamericana para la Investigación y el Desarrollo Educativo ISSN $2007-7467$

Figura 10. Comparación de calificaciones por programa educativo

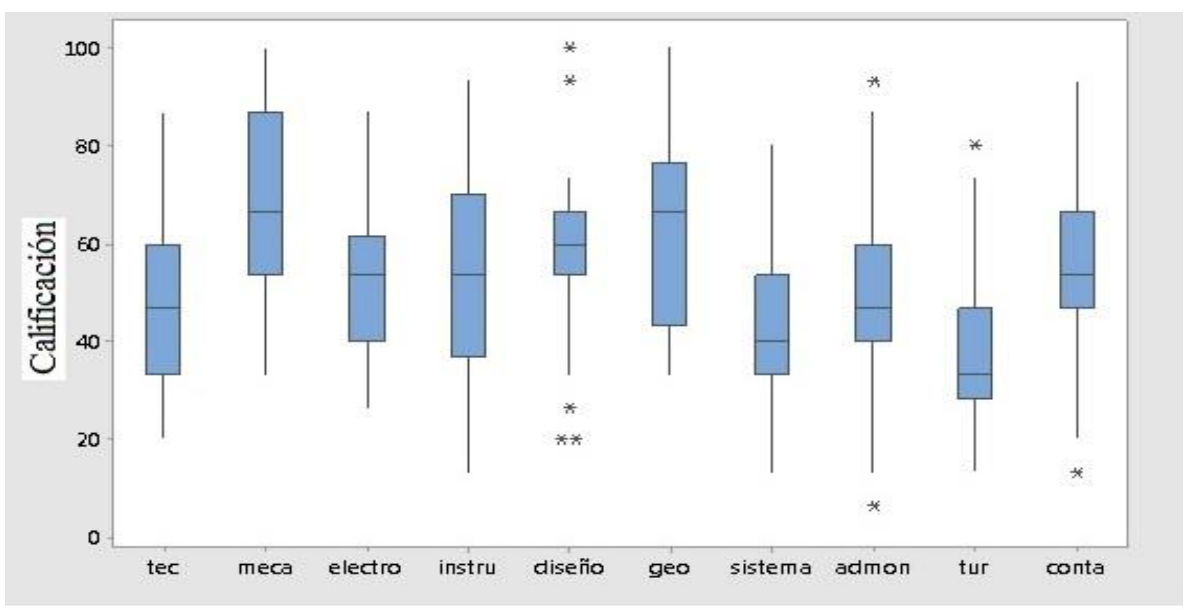

Fuente: Elaboración propia

La figura 10 muestra los diagramas de caja de cada programa educativo tomando la calificación. El programa educativo de ingeniería en Diseño Molecular de Materiales tiene menor rango intercuartil comparando con todos; tiene menor variabilidad de los datos porque la longitud de la caja es menor, aunque tiene cinco valores atípicos, ya que tiene dos valores de 20, un valor de 26.666, un valor de 93.333 y otro 100 .

Los programas con el valor mayor de la mediana $\left(Q_{2}=66.666\right)$ son ingeniería en Mecatrónica e ingeniería en Geofísica, pero aquella la tiene más cerca del primer cuartil $\left(Q_{1}\right)$, esto es, los datos superiores de la mediana están más separados hacia arriba o hacia el tercer cuartil $\left(Q_{3}\right)$, mientras en esta la mediana tiene más cercanía con el tercer cuartil $\left(Q_{3}\right)$, los datos inferiores a la mediana están más separados hacia abajo o hacia el $Q_{1}$. Estos dos programas educativos tienen el mismo valor mínimo (33.333) y el mismo valor máximo (100), sin ningún valor atípico.

Los programas educativos con igual mediana $\left(Q_{2}=53.333\right)$ son ingeniería en Instrumentación Electrónica y Nanosensores, Contaduría e ingeniería en Electrónica y Computación. En el caso de la ingeniería en Instrumentación Electrónica y Nanosensores se observa que la mediana está en medio del primer y tercer cuartil, esto significa que tiene igual cantidad de datos de la mediana hacia cada uno de los cuartiles $\left(Q_{1}\right.$ y $\left.Q_{3}\right)$. Mientras que en la ingeniería en Electrónica y Computación la mediana está más cerca del $Q_{3}$, los datos están sesgados hacia abajo (al $Q_{1}$ ). Por último, en la licenciatura en Contaduría la mediana está más cerca del $Q_{1}$, los datos están sesgados hacia arriba (al $Q_{3}$ ); este programa, además, tiene un valor atípico de 13.3333 . 


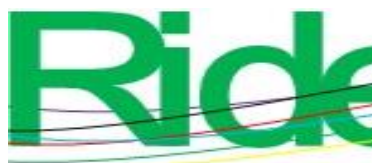

Revista Iberoamericana para la Investigación y el Desarrollo Educativo ISSN $2007-7467$

La licenciatura en Tecnologías de la Información y la licenciatura en Administración tienen igual valor de la mediana $\left(Q_{2}=46.666\right)$, pero la caja de aquella tiene mayor dispersión que la de esta última, y el primer cuartil es mayor en la licenciatura en Administración y la mediana de esta está más cerca del primer cuartil, los datos están sesgados al tercer cuartil. Además, este programa tiene dos valores atípicos, uno de 6.666 y otro de 93. El valor máximo de ambos que se tomó para formar el diagrama es 86.666 y el mínimo en la licenciatura en Administración es de 13.333 y en Tecnologías de 20; el tercer cuartil de ambos programas educativos es igual.

El programa educativo con menor mediana y menor rango intercuartil corresponde a la licenciatura en Turismo. Tiene un valor de la mediana de $Q_{2}=33.3333$, el valor del primer cuartil de $Q_{1}=28.3333$ y el valor del tercer cuartil es de $Q_{3}=46.666$.

Siguiendo los resultados descritos en este apartado, los hallazgos aquí encontrados son análogos a los encontrados por Ngo (2019), ya que este afirma que las brechas de habilidades que inhiben el acceso a cursos de nivel superior están relacionadas con fracciones, la resolución de ecuaciones algebraicas y la respuesta a problemas de palabras, los cuales se incluyeron en el instrumento que se aplicó a los estudiantes de primer ingreso del CUValles.

\section{Discusión}

El estudio se limitó a los programas educativos que llevan matemáticas en su plan de estudios, por lo que, para tener la visión completa del CuValles, podría valorarse la inclusión de los demás programas educativos que se ofrecen allí.

Por otra parte, el estudio solo consideró a los estudiantes de primer ingreso de un ciclo escolar, e igualmente podría ampliarse a otros semestres para verificar si los resultados tienen diferencias significativas.

Aunado a lo anterior, se podría dar seguimiento a los estudiantes considerados en el estudio en semestres posteriores, con la finalidad de identificar si mejoraron en sus conocimientos matemáticos.

Ahora bien, con el estudio se logró identificar los conocimientos en matemáticas con los que ingresan los estudiantes al CUValles, al igual que las carencias, lo cual es de gran importancia, tal y como lo afirman Ayebo, Ukkelberg y Assuah (2017), ya que con ello se puede tener un punto de partida para apoyarles en lo que requieren mejorar. 

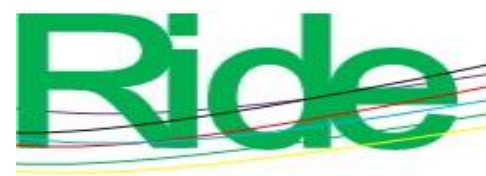

Revista Iberoamericana para la Investigación y el Desarrollo Educativo ISSN $2007-7467$

Entre las fortalezas del estudio podríamos señalar que este permitió identificar los conocimientos en matemáticas de los estudiantes de primer ingreso de los programas educativos que llevan cursos de matemáticas para implementar una propuesta didáctica a aquellos que requieren mejorar sus competencias en esta área. Por otra parte, también permitió, al igual que en Tenorio, Martín y Bermudo (2015), observar que los estudiantes carecen de conocimientos matemáticos básicos, los cuales debieron haber sido adquiridos en el bachillerato e incluso en la secundaria. En el mismo sentido, Ayebo et al. (2017) afirman que muchos de los estudiantes que ingresan a la universidad tienen mala preparación para las matemáticas que se estudian en la universidad. Consecuentemente, en este estudio se busca que los profesores de bachillerato conozcan qué es lo que se requiere que aprendan sus alumnos, qué necesitarán en la universidad, para que de esta manera se les pueda encaminar hacia esa dirección. La importancia del estudio, así como de los resultados obtenidos, van más allá de solo identificar los conocimientos matemáticos con los que ingresan los alumnos, ya que, siguiendo una vez más a Ayebo et al. (2017), uno de los grandes factores que influyen en que un estudiante esté o no preparado para su periplo universitario es el nivel de conocimientos en matemáticas que los estudiantes obtuvieron en el bachillerato, por lo que con este estudio se pueden tener elementos que atiendan la problemática identificada a nivel institucional. 


\section{Conclusiones}

De la información obtenida se puede deducir que los estudiantes que ingresan a las licenciaturas de Administración, Turismo, Contaduría Pública, Tecnologías de la Información y a las ingenierías en Mecatrónica, Electrónica y Computación, Instrumentación Electrónica y Nanosensores, Diseño Molecular de Materiales, Geofísica y Sistemas Biológicos en el CUValles de la Universidad de Guadalajara presentan deficiencias en conocimientos matemáticos básicos.

Los programas educativos que obtuvieron un promedio mayor al promedio general (52.97) fueron la ingeniería en Mecatrónica, en Instrumentación Electrónica y Nanosensores, en Diseño Molecular de Materiales, en Geofísica y la licenciatura en Contaduría Pública; el resto de los programas educativos tuvieron un promedio por debajo del promedio general.

Los estudiantes de ingeniería Mecatrónica y de Geofísica son los únicos que obtuvieron una calificación promedio aprobatoria, con 68.641 y 62.745 respectivamente. El resto de los programas educativos obtuvieron una calificación promedio reprobatoria.

Es necesario trabajar en los cursos de Matemáticas I y Precálculo, que los alumnos de primer ingreso de las licenciaturas antes citadas toman en el primer semestre, con el fin de subsanar las carencias en los conocimientos matemáticos básicos necesarios para el inicio de sus estudios. Particularmente, las áreas con mayor prioridad para su atención son: fracciones, exponentes, jerarquía de operaciones, ley de los signos y operaciones algebraicas en general.

Se considera de importancia relevante el compartir esta información con las escuelas de nivel medio superior de donde proceden los alumnos que ingresan a CU Valles, con el fin de que se orienten hacia la implementación de estrategias necesarias para mejorar estos resultados.

Por otra parte, con el fin de dar seguimiento a los avances logrados durante el primer ciclo escolar de los estudiantes en CUValles, es recomendable aplicar un examen inicial, así como uno final, para verificar si hay mejora en los conocimientos básicos de matemáticas, y de ser necesario emprender acciones que apoyen el desarrollo de los cursos de Matemáticas I y Precálculo. Es igualmente conveniente dar continuidad a este estudio durante varios semestres para tener una visión más clara de los errores más comunes de los alumnos, y para tomar, al mismo tiempo, las medidas adecuadas para su posible corrección. 


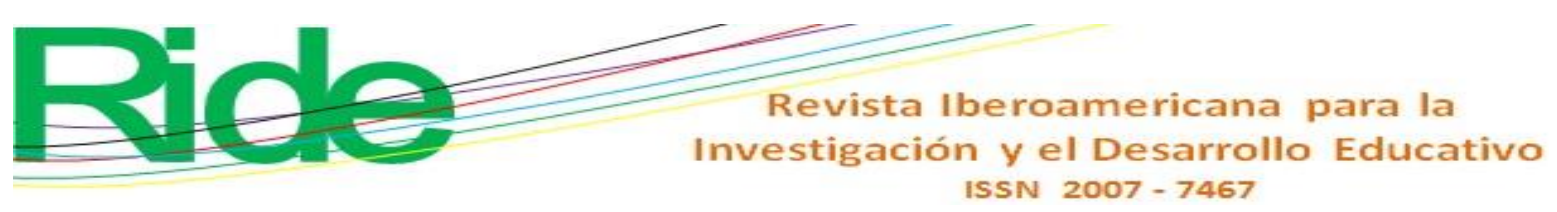

Ngo, F. (2019). Fractions in College: How Basic Math Remediation Impacts Community College Students. Research in Higher Education 60(4), 485-520. Retrieved from https://doi.org/10.1007/s11162-018-9519-x.

Stone, J. R. (2003). Improving Math Skills in CTE: How You Can Help. Techniques: Connecting Education \& Careers, 78(3), 58. Retrieved from https://search.ebscohost.com/login.aspx $?$ direct $=$ true $\& d b=t f h \& A N=9203716 \&$ site $=e$ host-live.

Stone, J. R., Alfeld, C. and Pearson, D. (2008). Rigor and Relevance: Enhancing High SchoStudents' Math Skills through Career and Technical Education. American Educational Research Journal, 45(3), 767-795. Retrieved from http://www.jstor.org/stable/27667150.

Teitelbaum, P. (2003). The Influence of High School Graduation Requirement Policies in Mathematics and Science on Student Course-Taking Patterns and Achievement. Educational Evaluation and Policy Analysis, 25(1), 31-57. Retrieved from https://doi.org/10.3102/01623737025001031.

Tenorio, A., Martín, A. y Bermudo, S. (2015). Alumnos de nuevo ingreso en ingeniería: un análisis de competencias matemáticas básicas. Épsilon - Revista de Educación Matemática, 32(1), 79-89.

Whannell, R. and Allen, B. (2012). First year mathematics at a regional university: Does it cater to student diversity? The International Journal of the First Year in Higher Education, 3(2), 45-58. Retrieved from http://dx.doi.org/10.5204/intjfyhe.v3i2.125. 


\begin{tabular}{|c|c|}
\hline & $\begin{array}{l}\text { Revista Iberoamericana para la } \\
\text { Investigación y el Desarrollo Educativo } \\
\text { ISSN } 2007-7467\end{array}$ \\
\hline Rol de Contribución & Autor (es) \\
\hline Conceptualización & $\begin{array}{l}\text { Silvia Sánchez Díaz «igual» Emilio Leonardo Ramírez Mora } \\
\text { «igual» César Calderón Mayorga «igual» } \\
\text { Rubén Sánchez Gómez «apoyo» }\end{array}$ \\
\hline Metodología & $\begin{array}{l}\text { Silvia Sánchez Díaz «igual» Emilio Leonardo Ramírez Mora } \\
\text { «igual» César Calderón Mayorga «igual» } \\
\text { Rubén Sánchez Gómez «apoyo» }\end{array}$ \\
\hline Software & $\begin{array}{l}\text { Silvia Sánchez Díaz «igual» Emilio Leonardo Ramírez Mora } \\
\text { «igual» César Calderón Mayorga «igual» } \\
\text { Rubén Sánchez Gómez «apoyo» }\end{array}$ \\
\hline Validación & $\begin{array}{l}\text { Silvia Sánchez Díaz «igual» Emilio Leonardo Ramírez Mora } \\
\text { «igual» César Calderón Mayorga «igual» Rubén Sánchez } \\
\text { Gómez «apoyo» }\end{array}$ \\
\hline Análisis Formal & $\begin{array}{l}\text { Silvia Sánchez Díaz «principal» Emilio Leonardo Ramírez } \\
\text { Mora «que apoya» César Calderón Mayorga «que apoya» } \\
\text { Rubén Sánchez Gómez «apoyo» }\end{array}$ \\
\hline Investigación & $\begin{array}{l}\text { Silvia Sánchez Díaz «igual» Emilio Leonardo Ramírez Mora } \\
\text { «igual» César Calderón Mayorga «igual» } \\
\text { Rubén Sánchez Gómez «apoyo» }\end{array}$ \\
\hline Recursos & $\begin{array}{l}\text { Silvia Sánchez Díaz «igual» Emilio Leonardo Ramírez Mora } \\
\text { «igual» César Calderón Mayorga «igual» } \\
\text { Rubén Sánchez Gómez «igual» }\end{array}$ \\
\hline Curación de datos & $\begin{array}{l}\text { Silvia Sánchez Díaz «igual» Emilio Leonardo Ramírez Mora } \\
\text { «igual» César Calderón Mayorga «igual» } \\
\text { Rubén Sánchez Gómez «apoyo» }\end{array}$ \\
\hline $\begin{array}{l}\text { Escritura - Preparación del } \\
\text { borrador original }\end{array}$ & $\begin{array}{l}\text { Silvia Sánchez Díaz «igual» Emilio Leonardo Ramírez Mora } \\
\text { «igual» César Calderón Mayorga «igual» } \\
\text { Rubén Sánchez Gómez «apoyo» }\end{array}$ \\
\hline $\begin{array}{l}\text { Escritura - Revisión y } \\
\text { edición }\end{array}$ & $\begin{array}{l}\text { Silvia Sánchez Díaz «igual» Emilio Leonardo Ramírez Mora } \\
\text { «igual» César Calderón Mayorga «igual» } \\
\text { Rubén Sánchez Gómez «apoyo» }\end{array}$ \\
\hline Visualización & $\begin{array}{l}\text { Silvia Sánchez Díaz «igual» Emilio Leonardo Ramírez Mora } \\
\text { «igual» César Calderón Mayorga «igual» } \\
\text { Rubén Sánchez Gómez «apoyo» }\end{array}$ \\
\hline Supervisión & $\begin{array}{l}\text { Silvia Sánchez Díaz «igual» Emilio Leonardo Ramírez Mora } \\
\text { «igual» César Calderón Mayorga «igual» } \\
\text { Rubén Sánchez Gómez «apoyo» }\end{array}$ \\
\hline
\end{tabular}




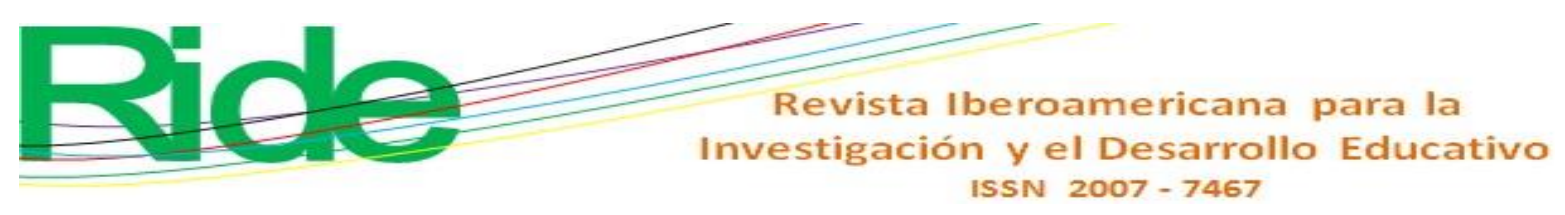

\begin{tabular}{|l|l|}
\hline $\begin{array}{l}\text { Administración de } \\
\text { Proyectos }\end{array}$ & $\begin{array}{l}\text { Silvia Sánchez Díaz «igual» Emilio Leonardo Ramírez Mora } \\
\text { «igual»César Calderón Mayorga «igual» } \\
\text { Rubén Sánchez Gómez «apoyo» }\end{array}$ \\
\hline Adquisición de fondos & $\begin{array}{l}\text { Silvia Sánchez Díaz «igual» Emilio Leonardo Ramírez Mora } \\
\text { «igual» César Calderón Mayorga «igual» } \\
\text { Rubén Sánchez Gómez «apoyo» }\end{array}$ \\
\hline
\end{tabular}

